\title{
The Theoretical Basis of Heat Flux Sensor Pen
}

\author{
XIAO DONG CHEN $\dagger$ \\ d.chen@auckland.ac.nz \\ Department of Chemical and Materials Engineering, The University of Auckland, \\ Private Bag 92019, Auckland City, New Zealand \\ SING KIONG NGUANG \\ sk.nguang@auckland.ac.nz \\ Department of Electrical and Electronic Engineering, The University of Auckland, \\ Private Bag 92019, Auckland City, New Zealand
}

\begin{abstract}
Measuring heat flux out or into a process surface is of significant practical interest. Miniaturization of the sensor and the capability of dynamic sensing are highly desirable. In this paper, the theoretical basis of a heat flux sensor 'pen' idea has been established, which allows for real-time measurements. Here the thin temperature measuring wires (thermocouple) are used as the heat flux sensing and heat carrier device. Such a device is shown to be feasible with the available solutions obtained using a semi-empirical approach employing Laplace transformation method combined with an intermediate curve-fitting procedure in the case of finite sensor length and exact solution already available in literature in the semi-infinite domain case.
\end{abstract}

Keywords: semi-infinite domain, Laplace transform, Heat flux

\section{Introduction}

Sensing heat flux at a working surface using a thermocouple wire setup is a recent advance in the dynamic gauging of mild frequency (in the order $10 \mathrm{kHz}$ or higher) processes like combustion engine operation [1]. By measuring the dynamics of the temperature change at the 'hard' surface of heat transfer, one can establish the instantaneous heat flux at this surface. However, the original contribution did not offer the basis for both low and high frequency processes. There was also a fundamental flaw where their technique derived for a finite thermocouple length was validated only for semi-infinite domain heat conduction [2]. In the paper by Reichelt, Meingast and Renz [1], the authors reported a means of calculating the wall heat flux from the time resolved measurements of the surface temperature. An analytical approach has been devised, based on an analytical solution

$\dagger$ Requests for reprints should be sent to Xiao Dong Chen, Department of Chemical and Materials Engineering, The University of Auckland, Private Bag 92019, Auckland City, New Zealand. 


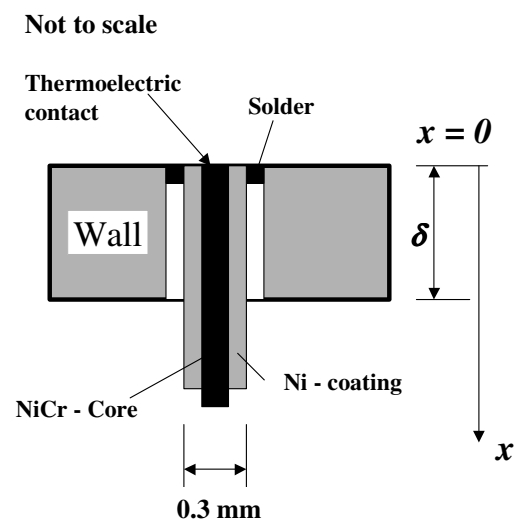

Figure 1. Schematic diagram of the set-up of the surface thermocouple (heat flux is experienced at the top surface)[1]

of the one-dimensional transient energy equation with transient boundary conditions using the Laplace transformation. The method was used to calculate the heat flux from a fuel spray to a wall at diesel engine conditions. The system of surface temperature measurement is shown schematically in Figure 1.

The thermocouple is soldered at its top to the wall and surrounded by an air gap. Because of this air gap, it was considered that the heat is conducted away from the wall along the thermocouple and one-dimensional equation can be used to describe this transfer of heat. To calculate the heat flux from the experimental measurements, this one-dimensional heat conduction equation needs to be solved analytically.

Upon examination of the air gap concept, it has been found that the air gap may not be sufficient to secure the one-dimensional assumption (see Appendix: Calculations). As such, a vacuum tube encasing the thermocouple is proposed in the following sections. Once the one-dimensional assumption is assumed to be satisfied, two scenarios are examined (i) finite thickness and (ii) Semi-infinite domain.

\section{The Concept of Heat Flux Sensor 'Pen'}

One version of the heat flux sensing device is given in Figure 2. The heat flux is desired to go through the wire along the symmetry of the air tube or 


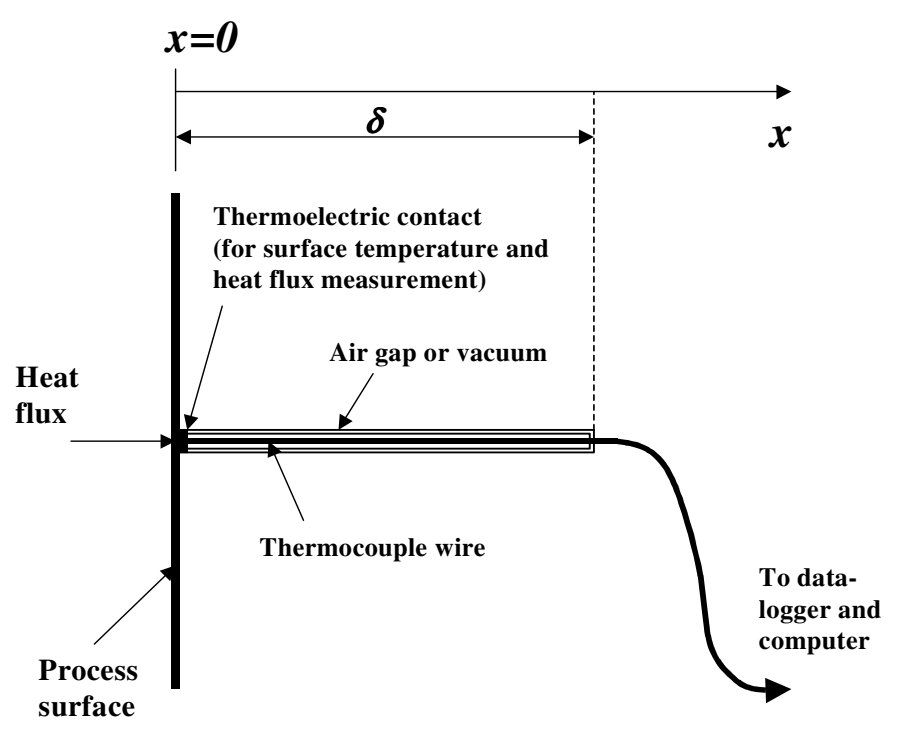

Figure 2. Schematic diagram of the set-up of the heat flux sensing pen

the vacuum tube. It can be shown that the vacuum tube would be ideal for insulating the wire to avoid radial heat loss. Air as an insulation medium is reasonable under certain conditions when the natural convection effect can be avoided (see sample calculations in the appendix). Because of the wire is used as the conduction medium it is possible to miniaturize the device.

\section{Two Case Studies}

(i) Finite length

The fundamental equation and the solution procedures are briefly outlined below and important points noted.

$$
\frac{\partial \theta}{\partial \tau}=\frac{\partial^{2} \theta}{\partial \xi^{2}} .
$$


Where the dimensionless parameters are:

$$
\tau=\frac{t \alpha}{\delta^{2}}, \quad \xi=\frac{x}{\delta}, \quad \theta=\nu-\nu_{\delta}
$$

$\nu_{\delta}(K)$ is the temperature at $x=\delta(m)$, i.e. the temperature of the thermocouple wire at the position flushed with the bottom surface of the wall. $\theta$ is still dimensional $(K)$.

The boundary conditions are:

$$
\begin{aligned}
& \theta(0, t)=\theta_{s}(t) \\
& \theta(1, t)=0
\end{aligned}
$$

In essence, there are two temperatures involved in calculating the heat flux, except is assumed to be constant throughout the experimental period. The initial condition is

$$
\theta(\xi, 0)=0
$$

The wall heat flux $\left(W \cdot m^{-2}\right)$ at top surface can be expressed as follows:

$$
\dot{q}^{\prime \prime}(\tau)=-\frac{\lambda}{\delta} \frac{\partial \theta}{\partial \xi}(0, \tau)
$$

Applying the basic principles of Laplace transformation to equation (1), combined with the boundary and initial conditions, Reichelt, Meingast and Renz [1] obtained the following solution of the temperature gradient at top surface $(x=0)$ :

$$
\frac{\partial \Theta}{\partial \xi}(0, s)=-s \Theta_{s}(s) \frac{1+e^{-2 p}}{p\left(1-e^{-2 p}\right)}
$$

where, $\Theta$ is the transformed temperature (the same as $\bar{\theta}$ used in the original work [1]) and $p$ is a transformation variable $\left(p^{2}=s\right)$. The term in the bracket in the denominator was expanded according to the following:

$$
\frac{1}{1-x}=1+\sum_{n=1}^{\infty} x^{n}, x<1
$$

This led to an approximate solution:

$$
\frac{\partial \Theta}{\partial \xi}(0, s)=-s \Theta_{s}(s)\left(\frac{1}{p}+2 \sum_{n=1}^{\infty} \frac{e^{-2 n p}}{p}\right)
$$

Finally, performing the Laplace inverse transformation on equation (8) and substituting this into equation (5), the following expression is obtained for the heat flux:

$$
\dot{q}^{\prime \prime}(\tau)=\frac{\lambda}{\sqrt{\pi} \delta} \int_{0}^{\tau} \frac{d \theta_{s}}{d \tau}\left(\tau^{*}\right) \frac{1}{\sqrt{\tau-\tau^{*}}}\left(1+2 \sum_{n=1}^{\infty} e^{-n^{2} /\left(\tau-\tau^{*}\right)}\right) d \tau^{*}
$$


A fundamental problem arises from the application of equation (7), which requires large $p$ (corresponding to the frequency of the temperature changes). Reichelt et al [1] investigated the situations with frequencies of the order of $1 \mathrm{kHz}$ or greater (see Figures 5 of the original paper). Indeed, equation (9) is not universally applicable for wall heat flux measurement, e.g. it is not applicable in the low frequency ( $p$ is small) regimes for example the heat exchanger fouling measurement where the frequency of temperature change is perhaps of the order of $0.1 \mathrm{~Hz}$. From equation (6), one can see two simple bounds for high and low frequency:

When $p \rightarrow 0$

$$
\frac{\partial \Theta}{\partial \xi}(0, s) \approx-s \Theta_{s}(s) \frac{1}{p}\left(\frac{1}{p}-1\right) .
$$

When $p \rightarrow \infty$

$$
\frac{\partial \Theta}{\partial \xi}(0, s) \approx-s \Theta_{s}(s) \frac{1}{p} .
$$

Both equation (10) and (11) can lead to analytical solutions of the wall heat flux respectively [2]:

When $p \rightarrow 0$

$$
\dot{q}^{\prime \prime}(\tau)=\frac{\lambda}{\delta} \theta_{s}(\tau)-\frac{\lambda}{\sqrt{\pi} \delta} \int_{0}^{\tau} \frac{d \theta_{s}}{d \tau}\left(\tau^{*}\right) \frac{1}{\sqrt{\tau-\tau^{*}}} d \tau^{*}
$$

When $p \rightarrow \infty$

$$
\dot{q}^{\prime \prime}(\tau)=\frac{\lambda}{\sqrt{\pi} \delta} \int_{0}^{\tau} \frac{d \theta_{s}}{d \tau}\left(\tau^{*}\right) \frac{1}{\sqrt{\tau-\tau^{*}}} d \tau^{*}
$$

Equation (12) appears to converge to a constant heat flux if the process becomes time-independent. Because the parameter $p$ directly reflects the frequency of temperature change, the order of magnitude of $1 \mathrm{kHz}$ is likely that it is accurate enough for calculating the heat flux from a fuel spray to a wall at diesel engine conditions as in the original paper [1]. In [1], the numerical implementation of (9) ignored the $\sum$ term thus effectively the solution bound (13) was used. The influence of $p$ is illustrated in Figure 3, which plots $\frac{1+e^{-2 p}}{1-e^{-2 p}}$ (Function (i)) in equation (6) against $p$. (Function (ii)) in equation (10) is also plotted against $p$, which describes the very low $p$ range very well. The small $p$ solution (ii) matches the original solution (i) well for $p<0.1$. Beyond $p$ of about 2.75, (i) simply reduces to unity (to within $1 \%)$.

As such, there is little doubt that the proposed method [1] is only applicable to high frequency processes. 


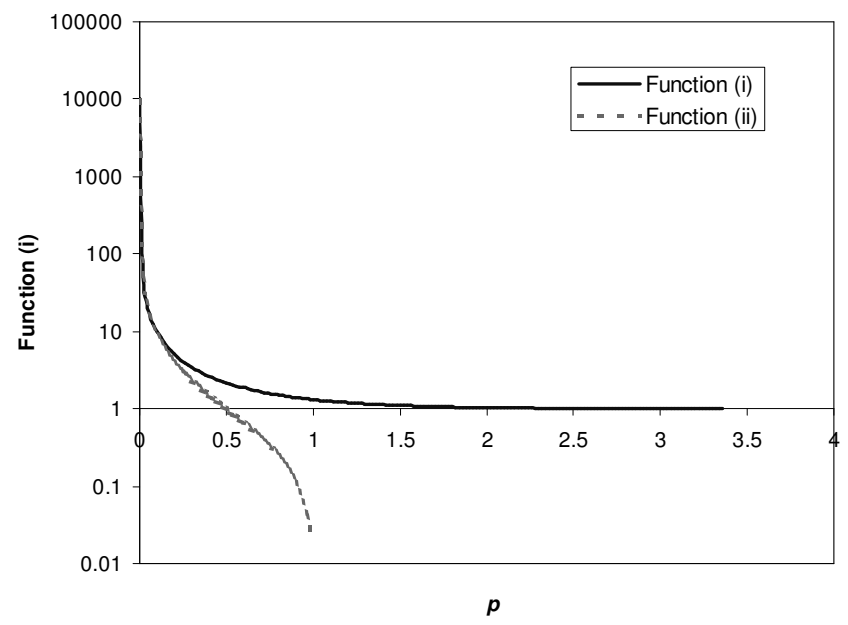

Figure 3. The effect of $\mathrm{p}$ value upon the Function (i) (also showing the goodness of the fit of Function (ii))

Now in order to obtain a solution structure that satisfy either the low frequency and the high frequency process as well as the intermediate situations, we have taken a piece-wise approach to approximate $\frac{1+e^{-2 p}}{1-e^{-2 p}}$, which then makes application of the above Laplace transformation possible (see Figure 4). When $p<4.16$

$$
\frac{1+e^{-2 p}}{1-e^{-2 p}} \approx 0.9524 p^{-1}-0.0018, r^{2}=1
$$

When $p \geq 4.16$

$$
\begin{gathered}
\frac{1+e^{-2 p}}{1-e^{-2 p}} \approx 0.0114 p^{-4}-0.1362 p^{-3}+0.5886 p^{-2}-0.1865 p^{-1}+1, \\
r^{2}=0.9998
\end{gathered}
$$

At the junction point between the two correlations (i.e. approximately at $p=4.16$ ) the slopes generated using these two equations has a discrepancy of only $1 \%$ while the absolute values predicted has a difference of $1.3 \%$. Practically, this would be of sufficient accuracy. Both equations (14 and $15)$ can be used to 'reverse' equation (6) back to the heat flux expressions, since [2] for $F(s)=1 / s^{n}(n=0,1,2, \cdots), f(t)=\frac{t^{n-1}}{(n-1) !}$ and for $F(s)=$ $1 / s^{v}(v>0), f(t)=\frac{t^{v-1}}{\Gamma(v)}$. 


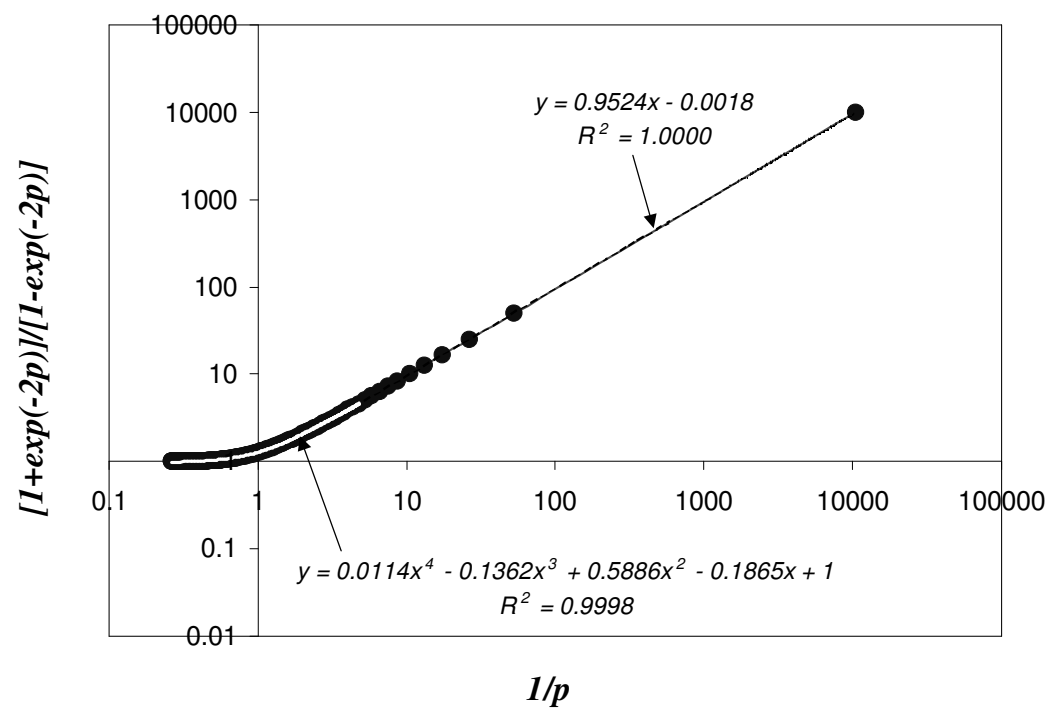

Figure 4. Piece-wise curve fits of the function for two regions of $\mathrm{p}$ (the solid circles are the exact values)

When $p<4.16$

$$
\dot{q}^{\prime \prime}(\tau) \approx 0.9524 \frac{\lambda}{\delta} \theta_{s}(\tau)-0.0018 \frac{\lambda}{\sqrt{\pi} \delta} \int_{0}^{\tau} \frac{d \theta_{s}}{d \tau}\left(\tau^{*}\right) \frac{1}{\sqrt{\tau-\tau^{*}}} d \tau^{*} .
$$

When $p \geq 4.16$

$$
\begin{aligned}
& \dot{q}^{\prime \prime}(\tau) \approx 0.1865 \frac{\lambda}{\delta} \theta_{s}(\tau)-\frac{\lambda}{\delta} \int_{0}^{\tau} \frac{d \theta_{s}}{d \tau}\left(\tau^{*}\right) \times \\
& \left(\frac{0.0114}{\Gamma\left(\frac{5}{2}\right)}\left(\tau-\tau^{*}\right)^{3 / 2}-0.1362\left(\tau-\tau^{*}\right)+\frac{0.5886}{\Gamma(3 / 2)}\left(\tau-\tau^{*}\right)^{1 / 2}+\right. \\
& \left.\frac{1}{\sqrt{\pi}}\left(\tau-\tau^{*}\right)^{-1 / 2}\right) d \tau^{*} .
\end{aligned}
$$

Noticeably, equation (17) has a 'linear' heat flux (or a 'DC' term) term (the 1 st term on the RHS) which presumably reduces its role as $\mathrm{p}$ in increases to infinity. Both the above equations can be solved numerically in the way similar to that presented in [1].

Although the finite length solutions are complete but in practice, the one-dimensional situation may not be realistic as the heat loss at radial direction cannot be assumed to be negligible (see Appendix for sample calculations). 
(ii) Semi-infinite domain

If the wire is long enough, the boundary condition at $x=\delta$ in equation (3) is no longer necessary. One now deals with a semi-infinite domain case. This, provided that the practicality allows the radial heat loss from the wire is negligible, makes the instrument more versatile as there is no need to ensure $\nu(\delta)$ to be a constant.

According to Carslaw and Jaeger [3], for semi-infinite domain $(x>0)$ with a boundary heat flux $f(t)$ at $x=0$, the following solution exists in dimensional form:

$$
\nu(x, t)=\frac{\alpha^{1 / 2}}{k \pi^{1 / 2}} \int_{0}^{t} f(t-\tau) e^{-\frac{x^{2}}{4 \alpha \tau}} \frac{d \tau}{\tau^{1 / 2}} .
$$

A numerical scheme can be set up to retrieve the heat flux from the temperature measurement at a location $x_{o}$. This location is preferred to be very close to the contact surface between the sensor tip and the heat transfer surface of concern (see Figure 2).

\section{Concluding Remarks}

It is feasible to use the formulas presented in this paper to construct a heat flux sensing pen. For the finite length of the thermocouple wire, the boundary conditions may be practically troublesome but the current study has nevertheless provided solutions for both low frequency and high frequency processes. With negligible heat loss from the wire, one can use the semi-infinite domain scenario to devise such a sensor. The solution procedure for the latter is straightforward.

\section{References}

1. L. Reichelt, U. Meingast, and U. Renz. Calculating transient wall heat flux from measurements of surface temperature. International Journal of Heat and Mass Transfer, 45:579-584, 2002.

2. J. L. Schiff. The Laplace Transform - Theory and Applications. Springer Verlag, New York, 1991.

3. H. S. Carslaw and J. C. Jaeger. Conduction of Heat in Solids, 2nd Ed. Clarendon Press, Oxford, 75-76,1959.

4. J. P. Holman. Heat Transfer, 4th Ed. McGraw-Hill, Tokyo, 1976.

5. F. P. Incropera and D. P. DeWitt. Fundamentals of Heat and Mass Transfer, 5th Ed. John Wiley \& Sons, New York, 2002. 


\section{Appendix A}

Sample Calculations on the Feasibility of the Heat Flux Sensor Pen

For a finite length of (cylindrical) wire (see Figure A.1), at steady state, the ratio of heat loss to the heat flow through the wire may be expressed as:

$$
\frac{Q_{\text {radial }}}{Q_{\text {axial }}}=\frac{h 2 \pi r \delta\left(\nu_{\text {ave }}-\nu_{\infty}\right)}{k \pi r^{2} \frac{\nu(0)-\nu(\delta)}{\delta}}=\frac{h \delta^{2}\left(\nu(0)+\nu(\delta)-2 \nu_{\infty}\right)}{k r(\nu(0)-\nu(\delta))}
$$

Considering the thermal conductivity $k=15 \mathrm{Wm}^{-1} \mathrm{~K}^{-1}[1,4]$ for the system shown in Figure 1 [1] for a wire diameter of $0.3 \mathrm{~mm}$, a typical temperature difference of $2 K$ between $x=0$ and $x=\delta$, and assuming the temperature at $x=\delta$ is the same as the ambient temperature $\nu(\delta)=\nu_{\infty}$, the above ratio has been calculated which illustrates the situations where heat loss becomes significant (say $>10 \%$ ). When this is significant, the heat flux sensing concept becomes inappropriate.

One example for bare wire like the one shown in Figure A.1 (as well as Figure 1) is given below:

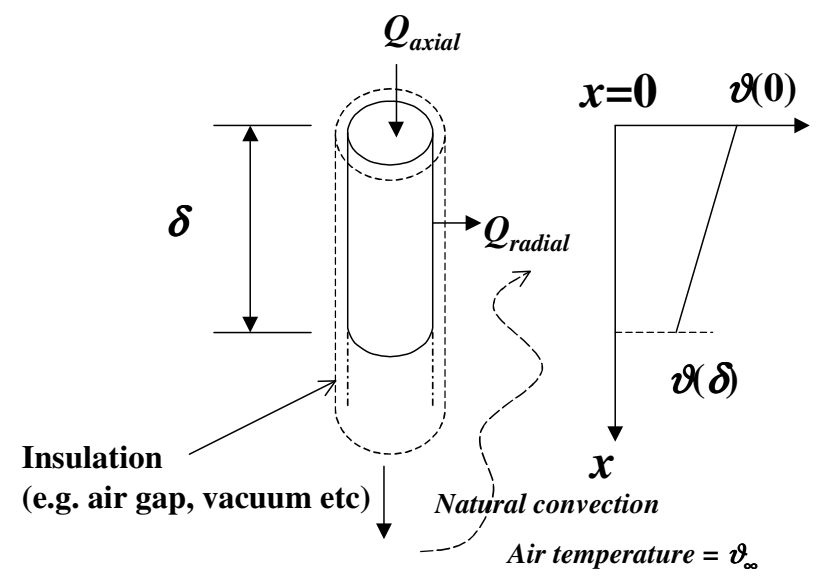

Figure A.1. Schematic diagram of the heat flux sensor system for heat loss ratio calculations. 
For natural convection, the lowest estimate of the heat transfer coefficient $h$ is about $5 \mathrm{Wm}^{-2} \mathrm{~K}^{-1}[4]$. Taking the working length of the wire as $\delta=1 \mathrm{~cm}$, equation (A.1) gives a ratio of 0.2 (meaning the heat lost over the $1 \mathrm{~cm}$ length to the ambient can be as high as $20 \%$ of the amount along the wire). This would make the set-up in Figure 1 highly questionable, as the one-dimensional heat conduction assumption would be invalid.

If one insulate the wire (refer to Figure 2) using the lowest conductivity layer (like a stagnant air shield or a vacuum tube (which may be filled with other insulating materials)), equation (A.1) is still valid except the heat transfer coefficient $\mathrm{h}$ should be replaced by the overall heat transfer coefficient $U$. $U$ can be calculated using the following equation [4].

$$
U=\frac{1}{\frac{r}{k_{i}} \ln \frac{r_{i}}{r}+\frac{r_{i}}{k_{o}} \ln \frac{r_{o}}{r_{i}}+\frac{r}{r_{o}} \frac{1}{h}}
$$

Here, the radius of the thermocouple wire is still labeled as $r . k_{i}$ and $r_{i}$ are the thermal conductivity of the material in the annuli (air or vacuum gap in Figure 2) and the radius of the inner side of the tube respectively. $k_{o}$ and $r_{o}$ are the thermal conductivity of the tube and the radius of the outer side of the tube respectively. $h$ is again the heat transfer coefficient due to natural convection.

For an air gap of $1 \mathrm{~mm}$ thick and the tube is made of glass of $1 \mathrm{~mm}$ thick, whilst other parameters are maintained the same as before, knowing that $k_{i}=0.024 \mathrm{Wm}^{-1} \mathrm{~K}^{-1}$ and $k_{o}=1.4 \mathrm{Wm}^{-1} \mathrm{~K}^{-1}, U$ can be found to be about $37 \mathrm{Wm}^{-2} \mathrm{~K}^{-1}$ inducing greater heat loss. This means that introducing an air gap insulator in the way shown in Figure 2 is not sensible. Furthermore, following the concept of 'critical insulation thickness' [5], a critical radius can be worked out using the following formula:

$$
r_{\text {crit }}=\frac{k_{i}}{h}
$$

giving $r_{\text {crit }}=0.024 / 5=0.0048 \mathrm{~m}$ (or $4.8 \mathrm{~mm}$ ). Below this radius, the air gap insulation can only increase heat loss rather than reduce, due to the well-known curvature effect.

Using vacuum or vacuum gap filled with solid insulating particles, the order of magnitude of the effective $k_{i}$ is $0.1 \mathrm{Wm}^{-1} \mathrm{~K}^{-1}$ or lower [4]. This gives a $U$ value of $0.32 \mathrm{Wm}^{-2} \mathrm{~K}^{-1}$. This would only lead to a radial heat loss of around $1.3 \%$ of that of the axial heat flow. This suggests that the vacuum seal would be appropriate for the measurement device suggested. Here the critical insulation radius is $0.02 \mathrm{~mm}$, which is within the radius of the thermocouple wire in Figure 1. 


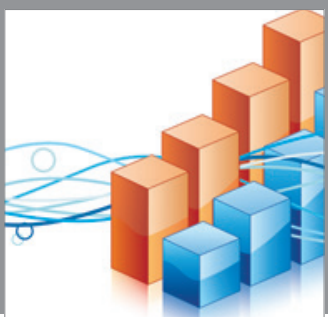

Advances in

Operations Research

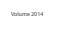

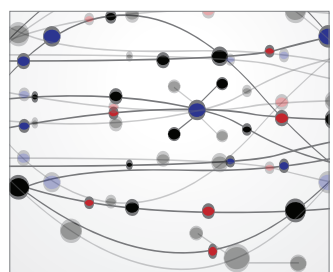

\section{The Scientific} World Journal
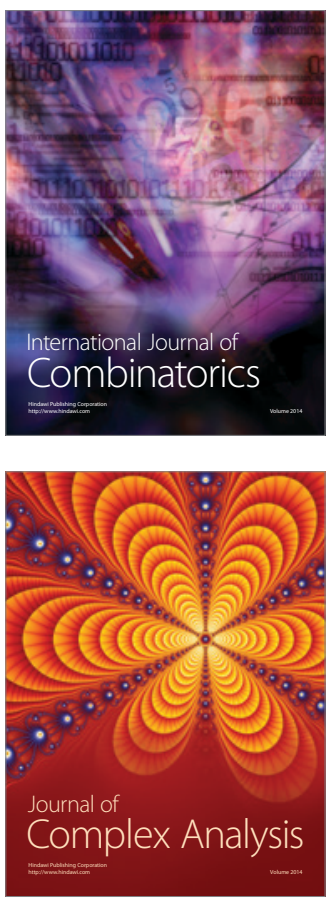

International Journal of

Mathematics and

Mathematical

Sciences
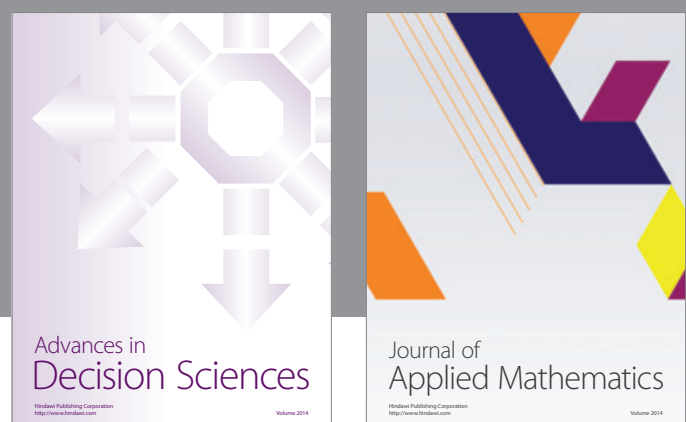

Journal of

Applied Mathematics
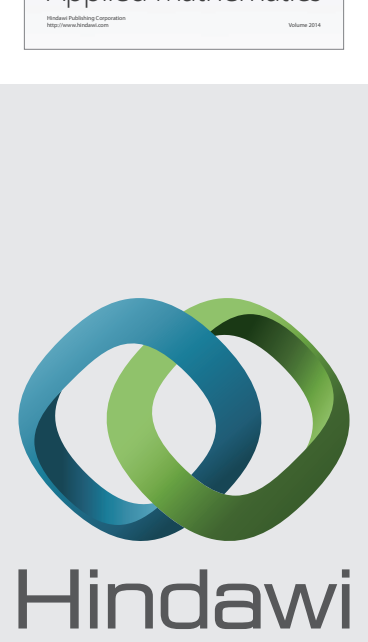

Submit your manuscripts at http://www.hindawi.com
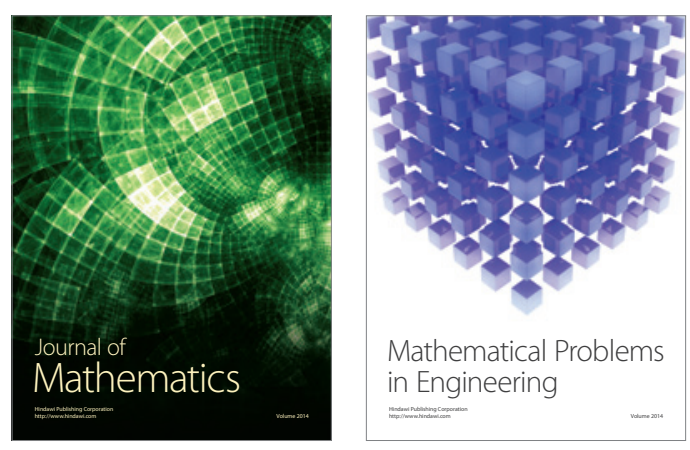

Mathematical Problems in Engineering
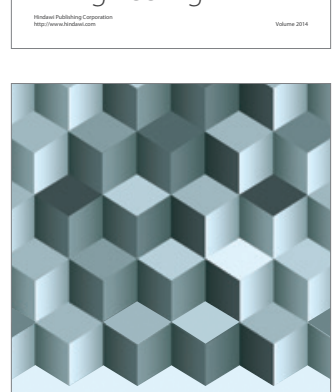

Journal of

Function Spaces
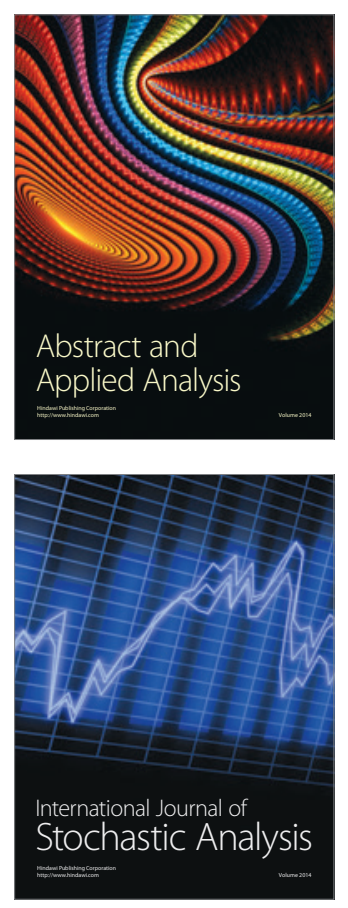

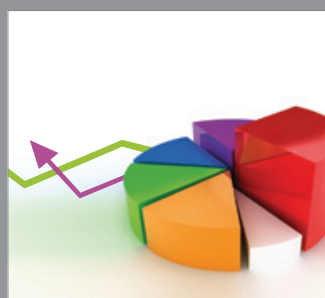

ournal of

Probability and Statistics

Promensencen
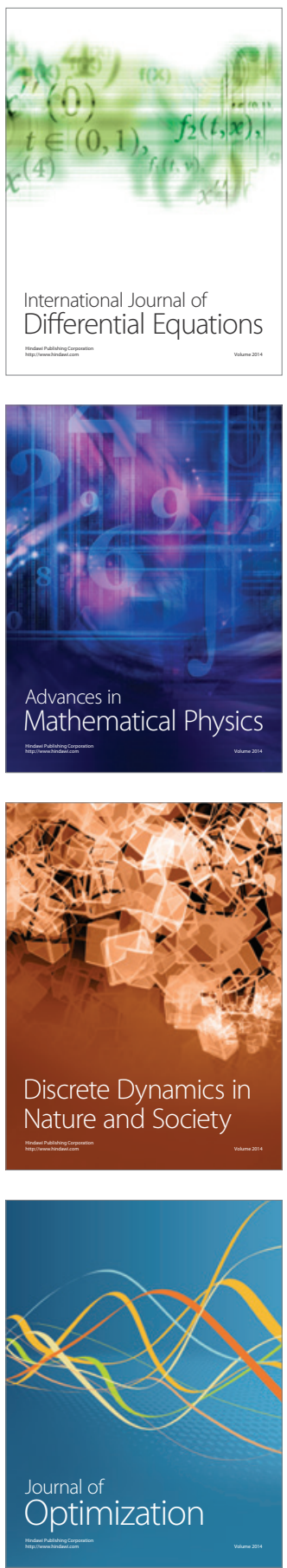